\title{
Avaliação da qualidade de vida de idosas com incontinência urinária: idosas institucionalizadas em uma instituição de longa permanência
}

\author{
Quality of life evaluation in elderly women with urinary incontinence: \\ institutionalized elderly women residents in a long-term institution

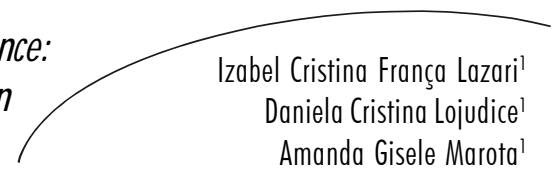

\section{Resumo}

A presente pesquisa tem como objetivo investigar a qualidade de vida de idosas com incontinência urinária, residentes em uma instituição asilar filantrópica do município de Catanduva, SP. Trata-se de um estudo descritivo, de corte transversal, cuja população foi composta de mulheres com idade igual ou superior a 60 anos, conscientes e orientadas no tempo e no espaço, capazes de interagir em uma entrevista. Foram excluídas as idosas que se recusaram a participar do estudo. A coleta dos dados foi feita através de entrevista com questionário composto por informações sociodemográficas e clínicas. $\mathrm{O}$ instrumento International Consultation on Incontinence Questionnaire Short From (ICIQ-SF) foi utilizado para avaliar a qualidade de vida das idosas incontinentes. A análise estatística dos dados foi feita em forma de média, desvio padrão, valores mínimos e máximos para cada variável. Encontrou-se um total de 22 idosas cuja média de idade foi de 72,9 anos +- 8,6. Todas as participantes relataram perda urinária, sendo que, destas, sete $(31,8 \%)$ relataram perder urina várias vezes ao dia. A maior porcentagem de perda urinária ocorreu antes de chegar ao banheiro (21,3\%), seguida de tosse ou espirro $(19,1 \%)$ e ao dormir $(12,8 \%)$. No que tange à interferência da perda urinária nas atividades de vida diária, a maioria (72,5\%) referiu alguma interferência. Concluiu-se que a incontinência urinária tem alta prevalência entre idosas institucionalizadas e compromete a qualidade de vida das mesmas. Diante disso, medidas de intervenção devem ser adotadas, na tentativa de minimizá-las ou combatê-las.

1 Instituto Municipal de Ensino Superior de Catanduva, Faculdade de Filosofia Ciências e Letras de Catanduva, Curso de Fisioterapia. Catanduva, SP, Brasil

Correspondência/Correspondence

Daniela Cristina Lojudice

Rua Corumbá, 411 - Jardim Salles

15804-345 - Catanduva, SP, Brasil

E-mail: daniela_lojudice@hotmail.com
Palavras-chave:

Meia-Idade. Idoso.

Mulheres. Inconti-

nência Urinária.

Qualidade de Vida.

Instituição de

Longa Permanência

para Idosos.

Catanduva, SP.

Epidemiologia

Descritiva. Estudos

Transversais. Saúde

da Mulher 
Abstract

This study aims to investigate the quality of life of elderly women with UI, all living in a philanthropic health care in Catanduva, S.P. It is a cross-sectional descriptive study whose population was women aged 60 years and more, oriented and conscious in time and space, able to be interviewed. The ones who refused to participate in the study were excluded. Data collection was done through a survey with a questionnaire composed of clinic and socialdemographic information. To evaluate the quality of life of incontinent elderly we used the (ICIQ-SF) short form-"International Consultation on Incontinence Questionnaire". The statistical data analisis comprised average, standard deviation, minimum and maximum values to each variable. We interviewed

Key words: Middle Aged. Aged. Women. Urinary Incontinence. Quality of Life. Homes for the Aged. Catanduva city. Epidemiology Descriptive. CrossSectional Studies. Women's Health 22 elderly women whose average age was 72,9 years old +- 8,6. All women reported urinary loss and seven $(31,8 \%)$ reported urinary loss several times a day. The higher percentage of urinary loss happened before they arrival to the bathroom $(21,3 \%)$, followed by coughing and sneezing $(19,1 \%)$ and sleeping $(12,8 \%)$. Concerning interference in the daily life activities, most of them $(72,5 \%)$ related some interference. We concluded that the Urinary Incontinence (UI) has high prevalence among institutionalized elderly and compromise their quality of live. So we believe that intervention measures should be taken in an attempt to reduce and fight then.

INTRODUÇÃO

A realidade brasileira demonstra que $o$ número de idosos dobrou nos últimos 50 anos, e as projeções indicam que no ano de 2020 esse segmento atingirá $15 \%$ da população nacional. ${ }^{1}$ De acordo com Ramos, ${ }^{2}$ no decorrer do envelhecimento, surgem limitações físicas, perdas cognitivas e doenças crônicas. Estas últimas podem ser consideradas uma ameaça à independência do indivíduo antes de um risco de vida. ${ }^{1}$

Uma das condições crônicas que mulheres e homens podem desenvolver, ao longo do envelhecimento, é a incontinência urinária (IU), sendo mais frequente entre o sexo feminino. ${ }^{3}$ De acordo com a Sociedade Internacional de Incontinência - Inter- national Continence Society, IU é definida como uma condição na qual ocorre perda involuntária de urina, sendo um problema social ou higiênico objetivamente demonstrado e interpretado, na maioria das vezes, como parte natural do envelhecimento, podendo levar a um quadro clínico de depressão, isolamento e vergonha, alterando, portanto, o convívio social. ${ }^{48}$

A porcentagem de IU entre mulheres jovens é de 10 a $20 \%$, e de 30 a $40 \%$ naquelas com idade igual ou superior a 65 anos. No Brasil, acredita-se que 11 a $23 \%$ da população feminina seja incontinente, embora muitas mulheres não relatem essa condição. ${ }^{5}$

A IU tem causa multifatorial, mas alguns fatores como o hipoestrogenismo, a multi- 
paridade, a idade avançada, cirurgias prévias, doenças predisponentes, assim como os defeitos pélvicos, contribuem para a perda da função esfincteriana. ${ }^{5,6}$ Além destes fatores, alterações decorrentes do envelhecimento, como a atrofia dos músculos e tecidos, queda funcional do sistema nervoso e circulatório e diminuição do volume vesical podem contribuir para o aparecimento da IU. Sendo assim, todos esses fatores, conjuntamente, contribuem com a redução da elasticidade e contratilidade da bexiga e causam a irritabilidade e instabilidade deste órgão. ${ }^{3}$

Apesar de não ser uma condição de vida ameaçadora, a IU pode causar vários problemas. ${ }^{9} \mathrm{Um}$ consenso na literatura demonstra que a incontinência urinária contribui para o surgimento de alterações psicológicas, de relacionamento pessoal e sexual, de relacionamentos sociais, bem como de alterações físicas e econômicas, influenciando negativamente a qualidade de vida dessa população.9-12

De acordo com Reis et al., ${ }^{6}$ a IU pode ser, na maioria dos casos, resolvida ou minorada diante de uma abordagem adequada.

A identificação dos fatores relacionados à incontinência urinária e o diagnóstico correto da sua etiologia são medidas importantes na adoção de recursos que almejam melhorar a qualidade de vida das mulheres incontinentes. ${ }^{4-6,9,12}$ Dessa forma, surgiu o interesse pelo presente trabalho, que tem como objetivo investigar a qualidade de vida de idosas com incontinência urinária, residentes em uma instituição asilar filantrópica do município de Catanduva, SP.

\section{CASUÍSTICA E MÉTODOS}

Trata-se de um estudo descritivo, de caráter transversal, realizado na instituição asilar filantrópica Associação São Vicente de Paulo, no período de janeiro a fevereiro de 2006.

A população do estudo foi constituída por todas as idosas ( 60 anos ou mais de idade) moradoras na instituição asilar já mencionada. As idosas tiveram que se apresentar conscientes e orientadas no tempo e no espaço, capazes de interagir em uma entrevista. Foram excluídas as idosas que se recusaram a participar do estudo e aquelas mulheres com idade inferior a 60 anos.

Inicialmente, as pacientes foram esclarecidas sobre a pesquisa a ser realizada e assinaram um Termo de Consentimento Livre e Esclarecido, segundo a Resolução no 196/ 96 do Conselho Nacional de Saúde. ${ }^{13}$

A coleta de dados foi feita mediante entrevista, utilizando um questionário que buscou informações referentes à identificação pessoal (iniciais do nome, data de nascimento, grau de escolaridade e estado civil). Em se tratando da utilização de medicamentos, também foi pesquisado o uso dos mesmos e a prática de fisioterapia. Em seguida, foi aplicado o instrumento International Consultation on Incontinence Questionnaire-Short Form (ICIQ-SF)". Esse questionário avalia a qualidade de vida de pacientes com incontinência urinária e contém informações referentes à frequência da perda urinária, quantidade de urina que é perdida, o quan- 
to que perder urina interfere nas atividades de vida diária, bem como o tempo e as circunstâncias da perda urinária. Nesse instrumento, há uma escala tipo Likert que avalia o quanto a incontinência urinária interfere na vida diária das pessoas, cuja pontuação varia de zero a dez, sendo que zero interfere pouco na vida do sujeito e dez, muito.

É um instrumento válido e confiável para mensurar a qualidade de vida de pessoas com incontinência urinária com alfa de Cronbach de 0,88. ${ }^{14}$ Foi originariamente desenvolvido e validado na língua inglesa por Avery et al. , $^{15}$ e validado para a realidade brasileira por Tamanini et al., ${ }^{14} \mathrm{em}$ estudo realizado em Hospital Universitário na cidade de Campinas, SP.

A análise estatística dos dados foi apresentada na forma de média, desvio padrão, valores mínimos e máximos para as variáveis numéricas. O programa Microsoft Windows 98 com Excel 1998 foi utilizado para a análise dos dados.

O presente projeto foi submetido à apreciação do Comitê de Ética em Pesquisa da Faculdade de Medicina de Catanduva, obtendo a aprovação em 09/01/2006.

\section{RESULTADOS}

Encontrou-se um total de 22 idosas. A média de idade entre as participantes foi de 72,9 anos, variando entre a idade mínima de 61 anos e a máxima de 90 anos, com desvio padrão de 8,6.
$\mathrm{Na}$ distribuição por faixa etária, verificou-se que dez idosas $(45,4 \%)$ tinham entre 60 a 69 anos, oito $(36,4 \%)$ entre 70 a 79 anos e apenas quatro $(18,2 \%)$ com 80 anos ou mais de idade.

De acordo com os dados referentes ao estado civil, verificou-se que a maioria das entrevistadas $(54,6 \%)$ era de solteiras, oito $(36,4 \%)$ eram de casadas e apenas duas $(9,1 \%)$ eram de viúvas.

A realização de fisioterapia por um período mínimo de três vezes semanais foi observada em apenas quatro idosas $(18,2 \%)$. A fisioterapia abordava exercícios de fortalecimento muscular, alongamentos, treinos de coordenação, de equilíbrio e de marcha.

O uso de medicamentos foi relatado por 19 idosas $(86,4 \%)$, todos receitados por médicos. Os medicamentos mais utilizados pelas idosas institucionalizadas foram os antipsicóticos (57\%), seguidos de anti-hipertensivos (32\%) e analgésicos (31\%).

Em relação à frequência de perda urinária das 22 idosas entrevistadas, sete $(31,8 \%)$ relataram perder urina diversas vezes ao dia, seis $(27,3 \%)$ perdiam duas ou três vezes durante a semana, cinco (22,7\%) perdiam urina o tempo todo e quatro $(18,2 \%)$ relataram perder urina uma vez ao dia.

A perda urináriade durante a realização de algumas atividades de vida diária (AVD) também foi pesquisada. Verificou-se que a 
maior porcentagem de perda urinária ocorreu antes de chegar ao banheiro $(21,3 \%)$, seguida de tosse ou espirro (19,1\%), como demonstrado na figura 1.

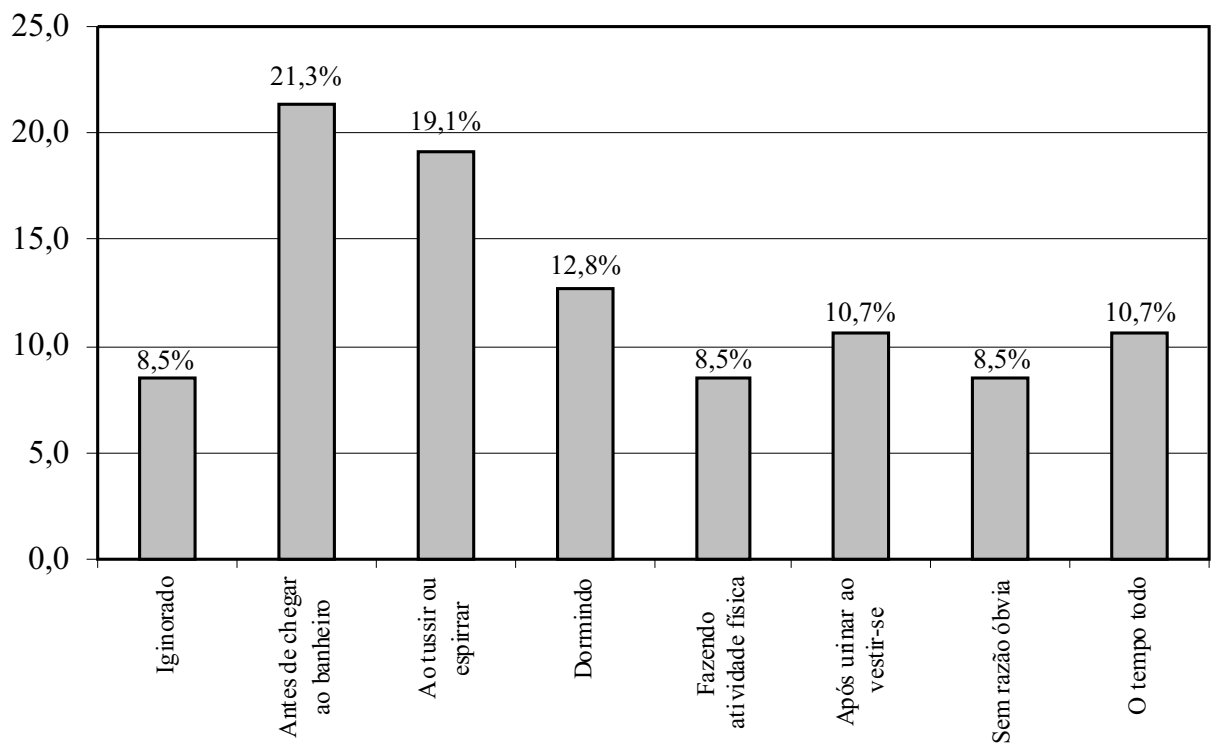

Figura 1 - Distribuição de perda urinária em relação às Atividades de Vida Diária. Catanduva, 2006.

Os dados obtidos em relação à interferência da perda urinária, nas atividades de vida diária das idosas institucionalizadas, podem ser observados na figura 2 . 


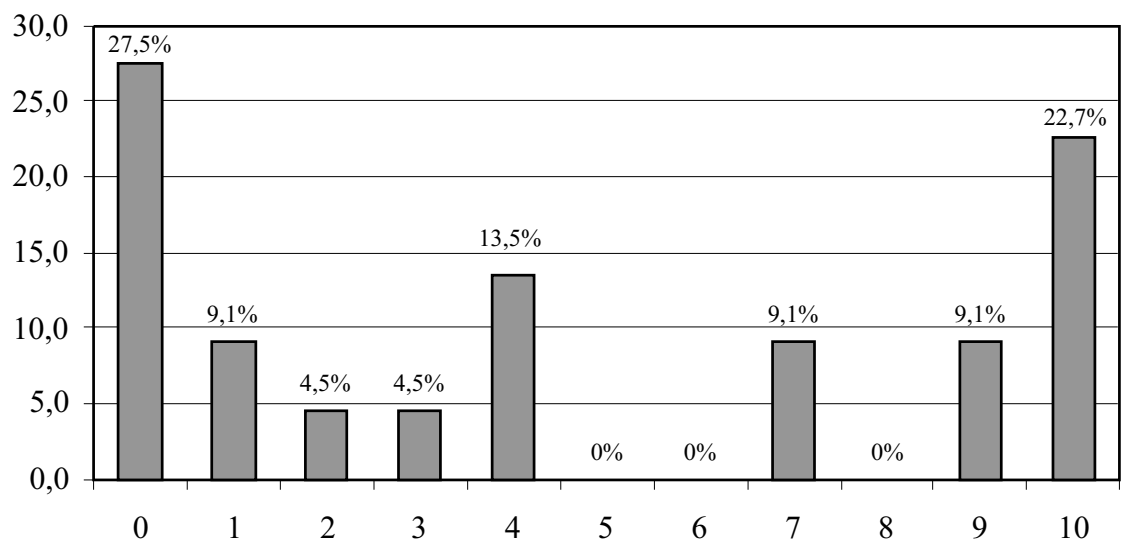

Figura 2 - Proporção da interferência da perda urinária na vida diária de idosas institucionalizadas. Catanduva, 2006.

Em relação à quantidade de perda urinária das 22 intrevistadas, nove idosas (40,9\%) relataram perdas pequenas, sete $(31,8 \%)$ perdas em grandes quantidades e seis $(27,3 \%)$ relataram perdas moderadas.

\section{DISCUSSÃO}

De acordo com a literatura, o envelhecimento da população brasileira tem ocorrido de forma rápida, com grande aumento na média de idade. No presente trabalho, verificou-se que a média de idade das idosas entrevistadas foi igual a 72,9 anos. Esse dado foi semelhante ao encontrado no estudo de Pereira. ${ }^{16}$

Em relação ao estado civil das participantes, os resultados da presente investigação revelaram que a maioria das idosas era de solteiras. Observações semelhantes foram observadas em outros trabalhos, como o de Moraes. ${ }^{17}$ Esse autor encontrou percentual majoritário para a categoria de solteiras entre mulheres acima de 60 anos, moradoras em asilo. Pereira ${ }^{16}$ relata que, aproximadamente, metade da população-alvo em seu estudo com idosos institucionalizados no Rio de Janeiro se enquadrava na categoria de solteiros, tendo destaque para o sexo feminino.

Embora o conhecimento dos benefícios em relação à prática de fisioterapia já tenha sido apontado, os idosos, independentemente de estarem institucionalizados ou domiciliados na comunidade, não se encontram inseridos em programas dedicados à prática de atividade física ou fisioterápica. Os dados do presente estudo demonstram que a maioria das idosas entrevistadas não realiza algum tipo de atividade física. Esse fato não parece estar restrito à instituição 
onde nossa pesquisa foi realizada, pois Feliciano, Moraes e Freitas ${ }^{18}$ verificaram em seu estudo que cerca de $83,0 \%$ dos idosos informaram não adotar essa prática, e a maior prevalência de sedentarismo ocorreu entre as mulheres que demonstraram dependência total ou parcial para as atividades de vida diária.

O uso de medicamentos foi relatado pela maioria das idosas asiladas (86,4\%). Semelhantes resultados foram apresentados por Lojudice. ${ }^{19}$ Essa autora verificou que 89,5\% dos idosos asilados faziam uso de tratamento medicamentoso e afirmou também que a maioria dos idosos faz uso de mais de um tipo de medicação simultaneamente. Tal informação é de extrema importância, pela interferência que medicamentos exercem na vida dos idosos, pois a farmacologia para esses indivíduos tem peculiaridades, em decorrência da redução de massa e diminuição da água corporal. Outras alterações que podem ocorrer incluem a diminuição na capacidade de filtração e excreção dos rins, bem como a queda do metabolismo hepático. Dessa forma, as ações farmacodinâmica e farmacocinética de um medicamento podem se dar de forma diferente nos idosos, contribuindo, portanto, para a ocorrência de dificuldade de eliminação de catabólitos, levando ao acúmulo de substâncias tóxicas e ao aparecimento de reações adversas. ${ }^{19}$ Embora este não tenha sido o direcionamento do presente trabalho, podemos afirmar que as drogas relacionadas ao controle da incontinência urinária são uma forma importante de tratamento conservador, mas as mesmas também podem desencadear reações adversas nos idosos.

Nas respostas específicas do questionário ICIQ-SF para a avaliação da incontinência urinária, verificou-se que das 22 participantes do estudo, sete $(31,8 \%)$ relataram diversas perdas urinárias ao dia e cinco $(22,7 \%)$ relataram perder urina duas ou três vezes por semana. De forma semelhante, Karantanis et al..$^{20}$ afirmaram que a frequência de perda de urina ocorreu em $87 \%$ das idosas que relataram perder urina sempre. Gunnel et al. ${ }^{11}$ verificaram que apenas $7 \%$ das pacientes entrevistadas relataram perda urinária pelo menos uma vez na semana.

Observamos que as maiores ocorrências de perda urinária se deram antes de chegar ao banheiro $(21,3 \%)$ e também ao tossir e espirrar (19,1\%). Karantanis et al. ${ }^{20}$ na pesquisa com o ICIQ-SF, também encontraram maior ocorrência de episódios quando as mulheres tossiam ou espirravam (55\%). Para Guarisi et al., ${ }^{21}$ de todas as pacientes por eles entrevistadas, $35 \%$ referiram perda urinária aos esforços, como tossir, rir e levantar peso. Os autores ainda salientam que a incontinência urinária tem interferência direta nas atividades diárias das idosas, de tal maneira que aquelas que são portadoras desta moléstia apresentam menores índices de qualidade de vida. No presente estudo, uma escala tipo likert foi utilizada para quantificar a interferência da perda de urina na vida das idosas. Em resposta a esta escala, o dado mais encontrado foi o de que a perda urinária nada significava $(27,5 \%)$, seguindo- 
se a resposta de grande interferência (22,7\%). Gunnel et al. ${ }^{11}$ encontraram um total de $86 \%$ de pacientes que não se sentiam restritas de suas atividades diárias e somente $14 \%$ declaram que a IU era um problema que as privava de realizar suas atividades sociais normais.

Nas respostas referentes à quantidade de perda urinária, nove idosas $(40,9 \%)$ relataram pequena quantidade de urina perdida e cinco $(22,7 \%)$ relataram grandes perdas. Esses dados estão de acordo com Karantanis et al. ${ }^{20}$ Independentemente da quantidade de perda urinária, a doença se torna estressante e debilitante, contribuindo para o isolamento social e depressão. ${ }^{8-14}$ Apresentase também como fator de risco para infecção urinária e distúrbio tegumentar. ${ }^{3,5}$ Além desses sinais e sintomas, a literatura ainda aponta que muitos pacientes vivem ansie- dade intensa por não estarem próximos a um banheiro, ou por medo de perder urina em público. ${ }^{5}$

\section{CONCLUSÕES}

Na presente investigação, constatou-se elevada ocorrência de incontinência urinária entre idosas residentes na instituição asilar estudada. Concluímos, também, que a presença da incontinência urinária interfere no estilo de vida das mesmas, contribuindo, portanto, para as alterações psicológicas e físicas e modificando o convívio com a comunidade onde estão inseridas e no grau de satisfação com a vida. Dessa forma, programas de intervenção devem ser adotados para minimizá-las ou combatê-las, melhorando, portanto, a qualidade de vida dessa população.

\section{REFERÊNCIAS}

1. Camarano AA. Envelhecimento da população brasileira: uma contribuição demográfica. In: Freitas EV, et al. Tratado de Geriatria e Gerontologia. Rio de Janeiro: Guanabara Koogan; 2002. p. 58-70.

2. Ramos LR. Epidemiologia do Envelhecimento. In: Freitas EV, Py L, Néri AL, Cançado FAX, Gorzoni ML, Rocha SM. Tratado de Geriatria e Gerontologia. Rio de Janeiro: Guanabara Koogan; 2002. p. 72-7.

3. Glashan RQ, Lelis MAS. Fatores de risco associados à incontinência urinária - é possível modificá-los. Acta paulista de enfermagem 1999; 12(1): 43-7.
4. Feldner-Junior PC, Bezerra LRPS, Girão MJBC, Castro RA, Sartori MGF, Baracat EC, et al. Valor da queixa clínica e exame físico no diagnóstico da incontinência urinária. Revista brasileira de ginecologia e obstetricia 2002; 24(2): 87-91.

5. Klüber L, Moriguchi EH, Cruz FBM. A influência da fisioterapia na qualidade de vida de mulheres com incontinência urinária: revisão. Revista Medica PUCRS 2002; 12(3): 243-9.

6. Reis RB, Cologna AJ, Martins ACP, Paschoalin EL, Tucci-Junior S, Suaid HJ. Incontinência urinária no idoso. Acta Cir Bras 2003; 18(5): 47-51. 
7. Rouhr G, Christensen K, Ulstrup K, Kragstrup J. Reproducibility and validity of simple questions to identify urinary incontinence in elderly women. Acta Obstet Gynecol Scand 2004; 83: 969-72.

8. Tamanini JTN, D'Ancona CAL, Botega NJ, Netto-Junior NR. Validação do "King's Health Questionnaire" para o português em mulheres com incontinência urinária. Rev Saúde Pública 2003; 37(2): 203-11.

9. Stach-Lempinen B, Sintonen H, Kujansuu E. The relationship between clinical parameters and health-related quality of life as measured by the 15D in incontinent women before and after treatment. Acta Obstet Gynecol Scand 2004; 83: 983-8.

10. Dallosso H, Matthews R, McGrother C, Donaldson $\mathrm{M}$. Diet as a risk factor for the development of stress urinary incontinence: a longitudinal study in women. Eur J Clin Nutr 2004; 58: 920-6.

11. Gunnel A, Jan-Erik J, Orjan G, Kerstin N. Urinary incontinence: prevalence, impact on daily living and desire for treatment. Scand J Urol Nephrol 2004; 38: 125-30.

12. Stach-Lempinen B, kirkinen P, Laippala P, Metsãnoja R, Kujansuu E. Do objective urodynamic or clinical findings determine impact of urinary incontinence or its treatment on quality of life? Urology 2004; 63: 67-72.

13. Brasil. Ministério da Saúde. Resolução 196/ 96 do Conselho Nacional de Saúde/MS sobre Diretrizes e Normas

Regulamentadoras de Pesquisa Envolvendo seres humanos. Diário oficial da União, 10 de outubro de 1996.

14. TamaniniJTN, Dambros M, D' Ancona CAL, Palma PCR, Netto-Junior NR. Validação para o português do "international consultation on incontinence questionnaire - short form" (ICIQ-SF). Rev Saúde Pública 2004; 38(3): 438-44.

15. Avery K, Donovan J, Abrams P. Validation of a neu questionnaire for incontinence: the international Consultation on Incontinence Questionnaire (ICIQ). Abstract n.86 of the International Continence Society 31 st annual meeting. Seoul, Korea. Neurol Urodynamics, 20:510-1, 2001; 20: 510-1.

16. Pereira RS. Risco de desnutrição em idosos institucionalizados do município do Rio de Janeiro: um estudo com a mini avaliação nutricional (MAN) [dissertação]. Rio de Janeiro: Universidade do Estado do Rio de Janeiro; 2004.

17. Moraes IAL. A saúde do idoso no contexto asilar: implicações da institucionalização e as ações interventivas de enfermagem [dissertação]. Rio de Janeiro: Faculdade de Enfermagem, Universidade do Estado do Rio de Janeiro; 2002.

18. Feliciano AB, Moraes AS, Freitas ICM. O perfil do idoso de baixa renda no Município de São Carlos, São Paulo, Brasil: um estudo epidemiológico. Cad Saude Publica 2004; 20(6): 1575-85.

19. Lojudice DC. Quedas de idosos institucionalizados: ocorrência e fatores associados [dissertação]. Ribeirão Preto: Faculdade de Medicina de Ribeirão Preto/ USP; 2005.

20. Karantanis E, Fynes M, Moore KH, Stanton SL. Comparison of the ICIQ-SF and 24-hour test with other measures for evaluating the severity of urodynamic stress incontinence. International Urogynecological Association 2004; 15:111-6.

21. Guarisi T, Pinto-Neto AM, Osis MF, Pedro AO, Paiva LHC, Faúndes A. Incontinência urinária entre mulheres climatéricas 
brasileiras: inquérito domiciliar. Rev Saude Publica 2001; 35(5): 428-35.

22. Guarisi T, Pinto-Neto AM, Osis MF, Pedro AO, Paiva LHC, Faúndes A.
Procura de serviço médico por mulheres com incontinência urinária. Revista brasileira de ginecologia e obstetricia 2001; 23 (7): 439-43.

Recebido: 04/6/2008

Aprovado: 03/11/2008 